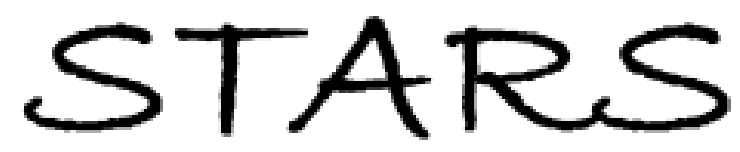

University of Central Florida

STARS

$1-1-2002$

\title{
Electrophoresis of an end-labeled polymer chain: A molecular dynamics study
}

\author{
Aniket Bhattacharya \\ University of Central Florida \\ Andrey Milchev \\ University of Central Florida
}

Find similar works at: https://stars.library.ucf.edu/facultybib2000 University of Central Florida Libraries http://library.ucf.edu

This Article is brought to you for free and open access by the Faculty Bibliography at STARS. It has been accepted for inclusion in Faculty Bibliography 2000 s by an authorized administrator of STARS. For more information, please contactSTARS@ucf.edu.

\section{Recommended Citation}

Bhattacharya, Aniket and Milchev, Andrey, "Electrophoresis of an end-labeled polymer chain: A molecular dynamics study" (2002). Faculty Bibliography 2000s. 3082.

https://stars.library.ucf.edu/facultybib2000/3082

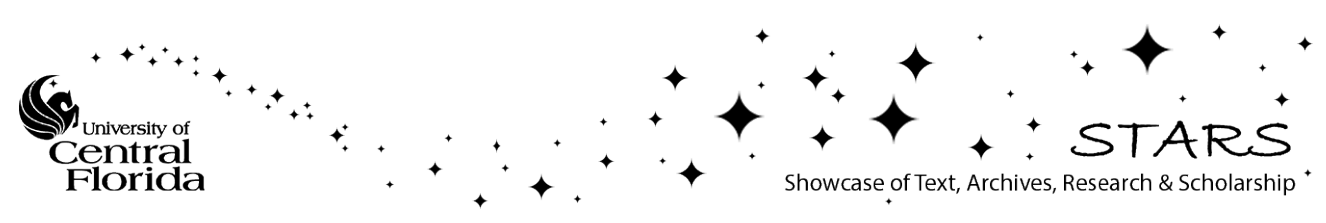




\title{
Electrophoresis of an end-labeled polymer chain: A molecular dynamics study
}

\author{
Aniket Bhattacharya ${ }^{1, *}$ and Andrey Milchev ${ }^{1,2}$ \\ ${ }^{1}$ Department of Physics, University of Central Florida, Orlando, Florida 32816-2385 \\ ${ }^{2}$ Institute for Physical Chemistry, Bulgarian Academy of Sciences, Georgi Bonchev Street, Block 11, 1113 Sofia, Bulgaria
}

(Received 7 June 2002; published 21 October 2002)

\begin{abstract}
We study the conformational and the dynamic properties of an end-labeled (telechelic) polymer chain embedded in a porous medium made of randomly distributed immobile spherical obstacles using a stochastic molecular dynamics (MD) simulation method for several obstacle densities $\rho_{\text {imp }}$ and for various field intensities $F_{x}$ applied only to one end of the chain. For $F_{x}=0$, the chain initially shrinks with increasing density of the obstacles $\rho_{i m p}$. In general, for small $F_{x}$ and low $\rho_{\text {imp }}$, the chain elongates along the direction of the force and shrinks in the transverse direction whereby this effect becomes more pronounced at larger chain lengths. However, we notice that for moderate values of $\rho_{i m p}$ and $F_{x}$, the conformational properties exhibit extrema before reaching a saturation at larger values of $\rho_{i m p}$ and $F_{x}$. Likewise, we also find that the drift velocity $V_{d}$ of the center of mass of the chain is a nonmonotonic function of the field intensity in the sense that $V_{d}$ also exhibits a maximum at a critical value of the field intensity $F_{x}^{\text {crit }}$ beyond which it decreases. Our MD results indicate that for large $\rho_{i m p}$ the chain still can be described by a self-avoiding random walk, which contradicts the prediction of variational calculation using the replica trick, but supports a more recent analytical result using the optimal fluctuation method, as well as a Monte Carlo simulation result for a slightly different disordered medium.
\end{abstract}

DOI: 10.1103/PhysRevE.66.041806

\section{INTRODUCTION}

The behavior of a polymer chain in random media [1,2] has gained considerable attention for more than a decade. At a fundamental level the study of a polymer chain in disordered media stands as a testing ground for the extension and applicability of the theory of reptation $[3,4]$. At the same time the flow property of a polymer chain can be immensely useful in understanding many important processes, e.g., length dependent fractionation of DNA via gel electrophoresis, complex polymer mixtures, hazardous waste in sea etc.

Quenched disorders have nontrivial effects on the dynamics and conformational properties of either a diffusing or a drifting polymer chain. It has been argued that disorder introduces transverse fluctuations, and for the center of mass of the chain, these transverse fluctuations are purely diffusive [5]. Much of the earlier works on polymers in random media have been primarily directed to test the predictions of reptation theory. For a Gaussian chain, both the Monte Carlo (MC) [6] calculations and analytic calculations using the replica trick [7] predict that in presence of quenched disorder the chain shrinks and the length dependence of the diffusion constant gets slower than what is predicted by the reptation theory $[3,4]$. The extension of these studies from a Gaussian chain to a self-avoiding chain adds another dimension to the problem. For a self avoiding chain, the competition between the strength of the excluded volume interaction $w$ and that of the disorder $v$ yields very interesting conformational and localization properties [8-10]. For a polymer chain of length $N$ embedded in porous media characterized by the impurity density $\rho_{i m p}$, the variational calculations using replica trick [8] predict three different regimes for the polymer conformations; while for $w>v, R_{g}^{2} \sim N^{1.2}$, for $w=v$, the chain ex-

*Corresponding author's email address: aniket@physics.ucf.edu
PACS number(s): 36.20.-r, 82.35.Rs, 07.05.Tp

ecutes ordinary random walk $R_{g}^{2} \sim N$, and for high impurity densities $(v>w)$, the chain is localized with $R_{g}^{2} \sim \rho_{i m p}^{2 / 3} N^{2 / 3}$.

The MC calculations by Muthukumar and Baumgartner $[9,10]$ are consistent with these results. On the contrary, a second analytic calculation using optimal fluctuation theory in terms of a small parameter predicts [11] that for large impurity densities the conformational properties of a chain are still described by a self-avoiding random walk. When a polymer chain moves through a random medium it encounters cavities of different lengths. As the radius of gyration $\left\langle R_{g}\right\rangle$ of the chain becomes comparable or larger than the mean pore size it encounters entropic barriers as it passes through bottle necks [10]. It has been argued [11] that replica calculations relying on a single variational parameter might not be adequate to describe a more complicated free energy landscape justifying the need for further work to settle some of these issues. The transport properties of a polymer chain through porous media $[9,12]$ reveal some unsettled issues as well. Earlier MC calculations suggest [9] that chain dynamics in a dense system can be different than reptation. But more recent $\mathrm{MC}$ investigation of diffusion of a polymer chain through a sea of frozen polymer chains by Yamakov and Milchev [12] indicates that reptation is still the dominant mode of polymer diffusion in random media.

The above discussions refer to the diffusive and conformational properties of polymers in random media. Motivated by practical problems, e.g., electrophoresis of biomolecules under an external field, these studies of neutral polymers have also been extended to polyelectrolytes where the entire chain is charged and driven by an electric field [1]. A special case of this variety is a telechelic polymer chain where only a block in either end of the chain is charged, and the rest of the chain is neutral. The conformational and drift properties of these end-labeled chains are of significant practical importance and have been studied previously analytically and by Monte Carlo methods [5,14-17]. In the absence of bias, es- 
timates of both the transverse and the longitudinal size of a stretched polymer chain can be obtained using simple scaling arguments $[3,18]$. Analytic results which have been obtained using optimal fluctuation method indicate that the transverse fluctuations of the center of mass of a polymer chain are similar to that of a Brownian particle and exhibit diffusive behavior [5]. These calculations also obtain the first order correction to the drift velocity due to impurities. A recent MC simulation indicates that in presence of the impurities, these scaling properties are weakly violated [15]. The simulation data also shows that there exists a critical value of the bias beyond which a "jamming effect" is observed [15].

The purpose of this paper is to examine the properties of an end-labeled telechelic polymer chain, where only the first monomer of the chain is influenced by an external force using a stochastic molecular dynamics simulation method [1921] and calculate the dynamic properties of the chain directly from the simulation as a function of bias and impurity density as well. There are some distinct advantages of carrying out molecular dynamics (MD), as opposed to MC in this particular problem. First of all the drift velocity and other dynamic properties can be obtained directly from the simulation and do not require any assumption. Secondly, for large bias the MC approach may suffer practical difficulties in implementing the Boltzmann factor $\exp (-\beta \Delta E)$. On the contrary, in MD for large bias one needs to go to smaller time steps which renders the calculations more expensive albeit feasible. As a by product we also study the conformational and the diffusive properties of the chain at zero bias. To the best of our knowledge, this is the first molecular dynamics study of the electrophoretic behavior of a polymer chain in presence of quenched disorder. This may also serve to check the results obtained through dynamic MC and other methods.

The key features of our calculations are as follows. For the zero bias case, we find that the radius of gyration $\left\langle R_{g}\right\rangle$ of the chain is a nonmonotonic function of the impurity density $\left(\rho_{\text {imp }}\right)$ exhibiting a minimum. This trend was not reported in the previous MC calculations [9], but has been observed in more recent MC calculations for a slightly different nature of the quenched disorder [13]. This indicates that the result has more general validity for a variety of disordered media. As a function of the increasing bias $F_{x}$ we observe a saturation effect in both the conformational and the dynamical properties of the chain. For moderate and large values of the impurity density, the geometrical properties, e.g., the radius of gyration $\left\langle R_{g}\right\rangle$, end to end distance $\left\langle R_{e}\right\rangle$ etc., either exhibit maxima or a saturation. But for large impurity density the chain still can be described by a self-avoiding random walk; this result contradicts the prediction of replica theory but is in agreement with a theory using a small parameter and MC work. We do not find any obvious scaling property for the longitudinal and transverse sizes of the chain in presence of disorder. Consistent with several MC $[13,15-17]$ results, we also find that the velocity of the center of mass as a function of the bias exhibits a peak. This observation can have potential use in electrophoresis in other related experiments where the filtering method depends of the mobility of the chains.

The organization of the paper is as follows: in the following section we discuss the method and simulation details. In
Secs. III and IV we present the statics and the dynamic properties of the chain. In Sec. V we summarize the results and discuss ongoing and prospective future work.

\section{MODEL AND NUMERICAL PROCEDURE}

The molecular dynamics method that we have implemented here is the same as that previously employed by one of us [20] and very similar to the method adopted by Grest and co-workers earlier [19]. To simulate a constant temperature ensemble, the monomers are coupled to a heat bath and the equations of motion read as

$$
\ddot{\vec{r}}=-\vec{\nabla} U_{i}-\Gamma \dot{\vec{r}}_{i}+\vec{W}_{i}(t),
$$

where $\Gamma$ is the monomer friction coefficient and $\vec{W}_{i}(t)$ that describes the random force of the heat bath acting on each monomer is a Gaussian white noise with zero mean satisfying the fluctuation-dissipation relation:

$$
\left\langle\vec{W}_{i}(t) \cdot \vec{W}_{j}\left(t^{\prime}\right)\right\rangle=6 k_{B} T \Gamma \delta_{i j} \delta\left(t-t^{\prime}\right) .
$$

The potential $U_{i}$ consists of two parts $U_{L J}$, and $U_{\text {chain }}$. Here $U_{L J}$ is a Lennard-Jones (LJ) potential acting between any two pair of monomers

$$
U_{L J}(r)=4 \epsilon\left[\left(\frac{\sigma}{r}\right)^{12}-\left(\frac{\sigma}{r}\right)^{6}-\left(\frac{\sigma}{r_{c}}\right)^{12}+\left(\frac{\sigma}{r_{c}}\right)^{6}\right], \quad r \leqslant r_{c},
$$

where $r_{c}$ is the cutoff distance beyond which the LJ interaction is set to be zero and $r=\left|\vec{r}_{i}-\vec{r}_{j}\right|$ and $\vec{r}_{i}, \vec{r}_{j}$ are the locations of the $i$ th and $j$ th monomers, respectively. The parameter $\epsilon$ is the LJ energy parameter and $\sigma$ is the LJ length parameter. The LJ interaction is a purely repulsive potential if the cutoff distance $r_{c}=2^{1 / 6} \sigma$, but has an attractive part when $r_{c}=2.5 \sigma . U_{\text {chain }}$ is the finite-extendable nonlinear elastic (anharmonic spring) potential acting between pairs of successive monomers along a chain:

$$
U_{\text {chain }}(r)=-k \ln \left[1-\left(\frac{l}{l_{0}}\right)^{2}\right],
$$

in which $k$ is the energy parameter of the potential, $l$ is the distance between two neighboring monomers of the same chain, and $l_{0}$ is a length parameters which describes the extension range between two successive monomers. We have chosen $k=30$ and $l_{0}=1.5$ which make chain crossing practically impossible [19]. We use the reduced units throughout this study. So the unit of time is $\sigma(m / \epsilon)^{1 / 2}$ and the unit of temperature is $\epsilon / k_{B}$ where $k_{B}$ is the Boltzmann constant. The interaction of the polymer chain with the randomly placed impurities is described by the repulsive part of the LJ interaction. A constant force is added to the $x$ component of the force on the first monomer to simulate a telechelic chain.

We have integrated the equations of motion following an accurate scheme developed by van Gunsteren and Berendsen [21] which uses a bivariate distribution of Gaussian random numbers for the stochastic forces [22]. A very fast Gaussian 
random number generator [23] and a link-cell-list [24,25] for calculating the forces help to make the integration quite efficient. Time steps for most of the cases were chosen to be $(\Delta \tau)$ of 0.01 (in reduced units) which produced stable integration at temperatures of interest. For very large value of the external force and especially for large chains $\Delta \tau$ $\sim 0.0025$ was necessary which rendered the calculations for $N=64$ very time consuming. The calculations were carried out on a ten processor $(1 \mathrm{GHz})$ Linux cluster and required few months of continuous operation.

\section{RESULTS}

The simulations are carried out for chain lengths $N=8$, 16,32 , and 64 and for many different combinations of porosity (up to 0.6) and bias which renders these calculations very time consuming. For chain lengths 8,16 , and 32, the maximum bias that we apply is 5 and requires a time step $\Delta \tau=0.004$ for the algorithm to be stable. For $N=64$ it becomes very difficult to increase the bias beyond 2 even with a time step $\Delta \tau=0.0025$. Here we present the main results while more detailed and quantitative informations will require more computer time and will be reported later. The results that are shown here are averaged over 500-1000 initial configurations of the impurities. The error bars for the static quantities are very small and within the width of the symbols in the figures; however, the calculations of the drift velocity require a very large number of independent runs.

In Fig. 1 we show two snapshots of a chain of length 64 representing different typical conformations of a chain drifting through the porous medium which determine essentially both static and dynamic electrophoretic behavior. Figure 1(a) corresponds to a case when the head is stuck and the tail (on which no external field is exerted) has overtaken it, and Fig. 1(b) shows a freely drifting chain with the head pulling the rest of the chain.

\section{A. Static properties of the chain: Zero bias}

First we show the results for the zero bias case and compare our numerical results with those obtained from the analytic theories, as well as previous MC results [12]. Figure 2 shows the radius of gyration $\left\langle R_{g}\right\rangle$ as a function of impurity density $\rho_{\text {imp }}$ for chain lengths $N=8,16,32$, and 64 , respectively. The ubiquitous feature is that the average size of the chain initially decreases to a minimum value $R_{g}^{\min }$ at $\rho_{i m p}^{\min }(N)$ with increasing impurity density. The position of $\rho_{i m p}^{\min }(N)$ is a function of chain length $N$ and shifts to a lower value for longer chains. Similar result was found by Yamakov and Milchev for a different system where the disorder was made of initially relaxed and subsequently frozen network of polymer chains. Since all the interactions considered here are purely repulsive, the origin of the observed minimum is due to the entropic traps and barriers [10]. Imagine a situation where a chain is fully confined in a particular cavity connected to other neighboring cavities through bottle necks. In this case the chain under consideration encounters entropic barriers to wander in to the neighboring cavities as explained by Muthukumar and co-workers. Now imagine the
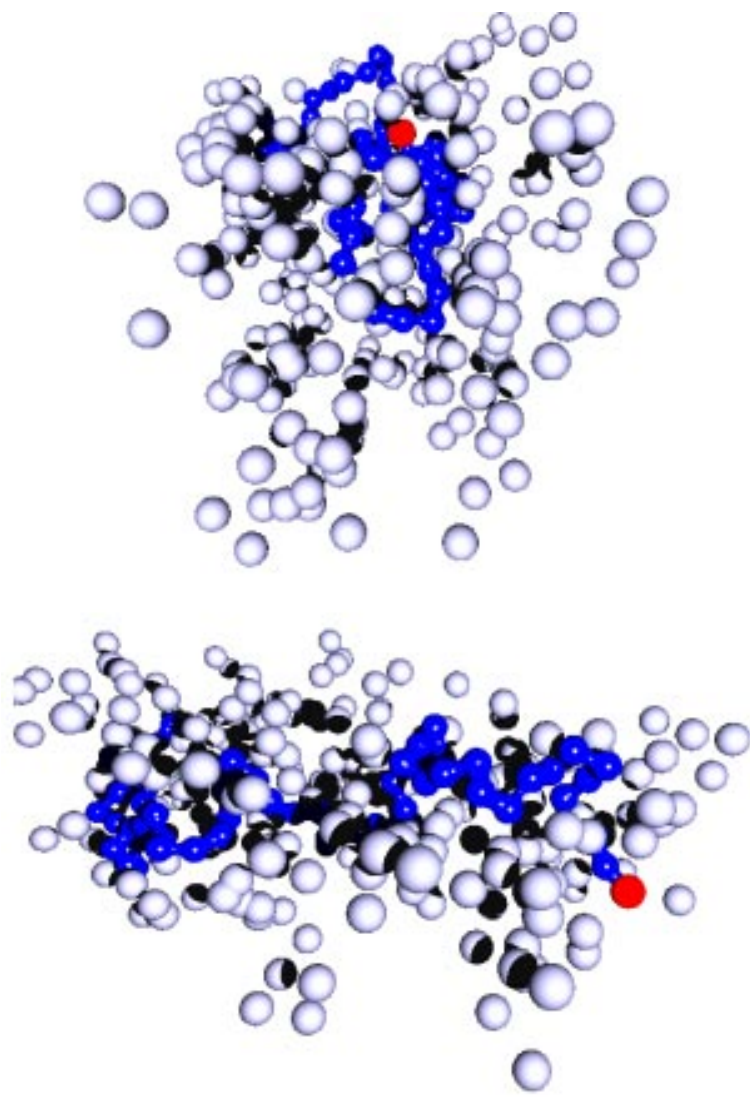

FIG. 1. The snapshots of two different conformations of a telechelic chain of length $N=64$ in porous media with $\rho_{i m p}=0.1$. In both cases the external field points from left to the right. The upper snapshot shows a situation where the rest of the chain has overtaken the immobile head which has got stuck between obstacles. The lower snapshot displays a freely drifting chain among obstacles. Most of the obstacles have been removed for better visibility.

impurities distances are scaled down without affecting the chain so that the average pore size becomes smaller. If the chain still could be accommodated in this particular pore it will have a smaller $\left\langle R_{g}\right\rangle$. This continues until $\rho_{\text {imp }}$ reaches $\rho_{\text {imp }}^{\text {min }}$. For larger $\rho_{\text {imp }}$, the average size of the cavities can not accommodate the full chain and the chain stretches in between the cavities with an increased $\left\langle R_{g}\right\rangle$ as alluded in the work of Panyukov [11]. This qualitatively explains the origin of the minimum in Fig. 2. For very long chains, this feature will be present only at extremely low impurity densities and therefore, may not be observed in the simulation.

We have calculated the effective Flory exponent $\nu_{\text {eff }}$ for various values of the impurity density $\rho_{i m p}$ as shown in Fig. 3(a). Evidently, at certain obstacle density $\rho_{\text {imp }}$ the medium creates random channels, characterized by "persistent length" which exceeds the persistent length of the polymer chain and effectively rectifies it thus leading to larger values of $\nu_{\text {eff }}>0.6$. At still larger obstacle concentration, some channels are interrupted and the polymer is confined in the cavities of comparable size which suppress its extension. Figure 3(a) shows that the chain may still be described by a self-avoiding random walk for large values of the impurity density. 


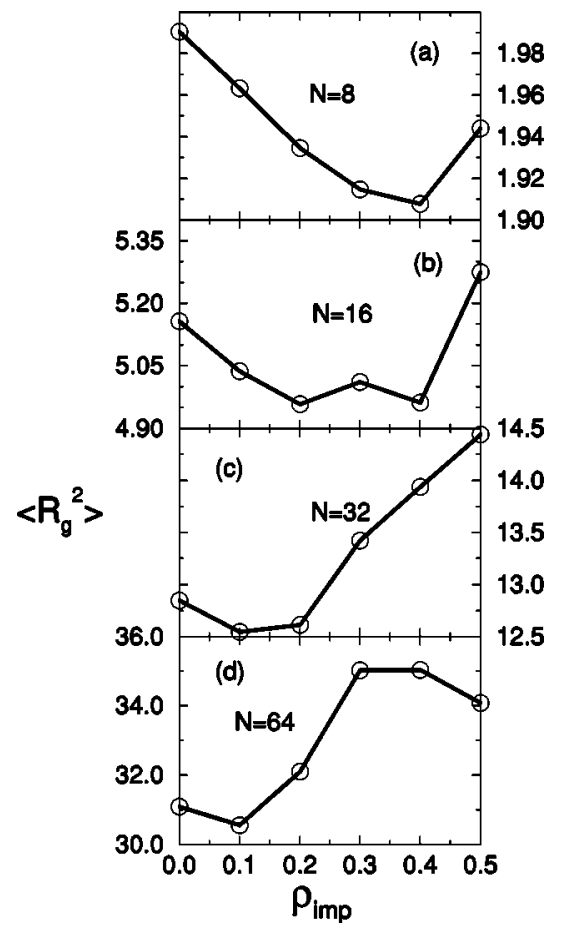

FIG. 2. $\left\langle R_{g}\right\rangle$ at zero external field as a function for different values of $\rho_{\text {imp }}$ for chain lengths (a) $N=8$, (b) $N=16$, (c) $N=32$, and (d) $N=64$, respectively. The characteristic minimum occurs at a lower value of the porosity as the chain length is increased.

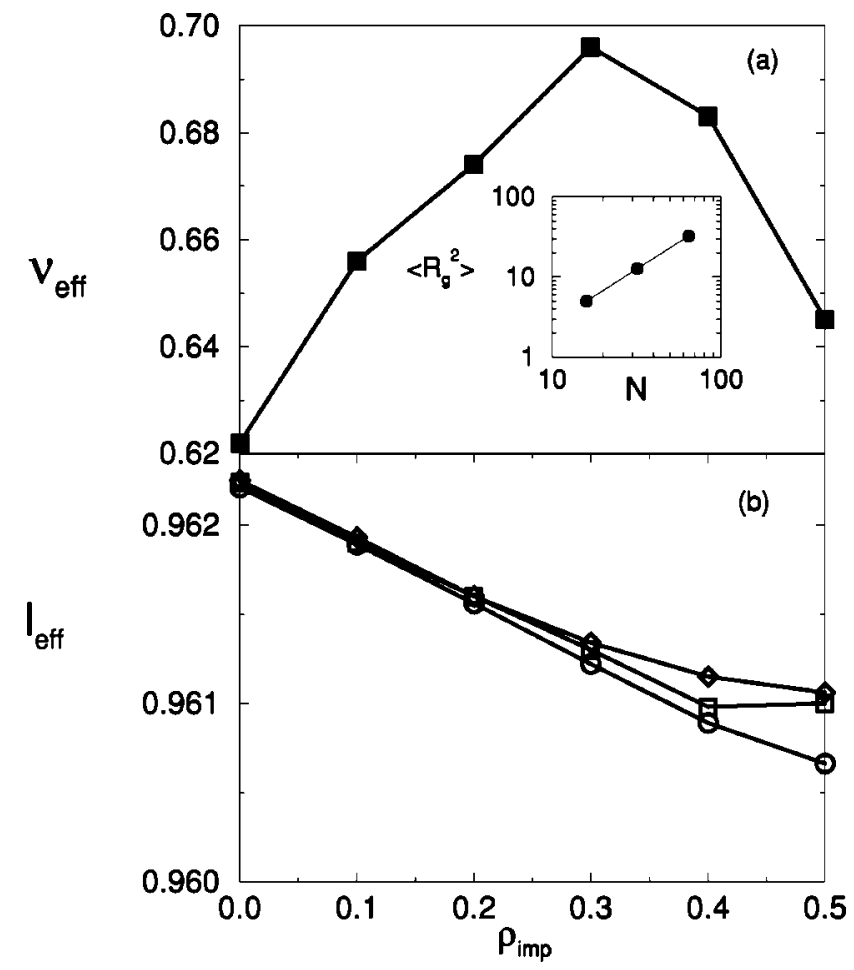

FIG. 3. The effective Flory exponent $\nu_{\text {eff }}$ and the renormalized bond length $l_{\text {eff }}$ as a function of the impurity density $\rho_{\text {imp }}$ for chain lengths $N=16,32$, and 64 , respectively.

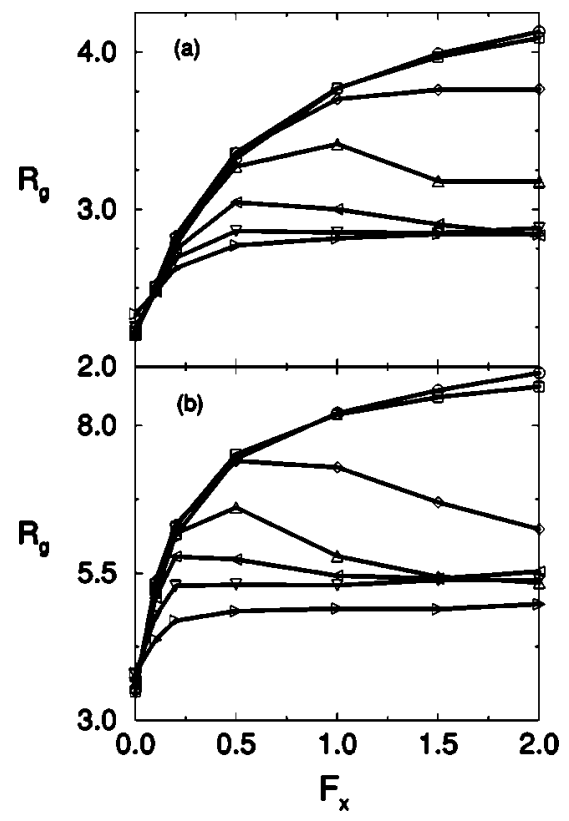

FIG. 4. $\left\langle R_{g}\right\rangle$ as a function of the bias $F_{x}$ for different values of $\rho_{\text {imp }}$; (a) for chain length $N=16$, (b) for chain length $N=32$, respectively. $\bigcirc, \square, \diamond, \triangle, \triangleleft, \nabla$, and $\triangleright$ correspond to $\rho_{i m p}$ $=0.0,0.1,0.2,0.3,0.4,0.5$, and 0.6 , respectively.

We have also monitored the average bond length between adjacent monomers as a function of $\rho_{i m p}$ which shows that the adjacent monomers are squeezed compared to their length without impurities; the average bond length decreases in general as $\rho_{\text {imp }}$ increases and tends to saturate at larger density. The relative squeezing is less for longer chain lengths as shown in Fig. 3(b). The change of bond length is very small and therefore can be neglected for the subsequent discussion.

\section{B. Static properties of the chain: Nonzero bias}

Now we look at the static properties of the chain in presence of a bias. Figures 4(a) and 4(b) show the typical conformational behavior of a chain, where we have plotted $\left\langle R_{g}\right\rangle$ as a function of the external field for different densities of the obstacles. For low densities of the obstacles, $\left\langle R_{g}\right\rangle$ increases with increasing bias without any signature of saturation. For high densities of the obstacles $\left\langle R_{g}\right\rangle$ becomes very quickly insensitive to the bias. For moderate densities we find that $\left\langle R_{g}\right\rangle$ exhibits a maximum and decreases subsequently. We believe that this is a generic behavior of the chain in a random media in presence of a bias. The peak position is a function both of the chain length and the bias. Similar qualitative behavior is observed for the end-to-end distance $\left\langle R_{e}\right\rangle$ and for the ratio $\left\langle R_{e}^{2}\right\rangle /\left\langle R_{g}^{2}\right\rangle$. In Fig. 5 we compare $\left\langle R_{e}^{2}\right\rangle /\left\langle R_{g}^{2}\right\rangle$ for a fixed density of the obstacles $\left(\rho_{\text {imp }}=0.2\right)$ as a function of the bias. It is evident that the longer the chain is, the more sensitive it is to the external field. Even for a tiny bias the longer $(N=64)$ chain has a significantly larger value of the ratio $\left\langle R_{e}^{2}\right\rangle /\left\langle R_{g}^{2}\right\rangle$ compared to its value in absence of the bias. The ratio $\left\langle R_{e}^{2}\right\rangle /\left\langle R_{g}^{2}\right\rangle$ lies in between 6 and 12 for a Gaussian chain and a fully stretched chain. We notice that for $N=64$ the maximum value of the ratio $\left\langle R_{e}^{2}\right\rangle /\left\langle R_{g}^{2}\right\rangle \sim 11$. Therefore 


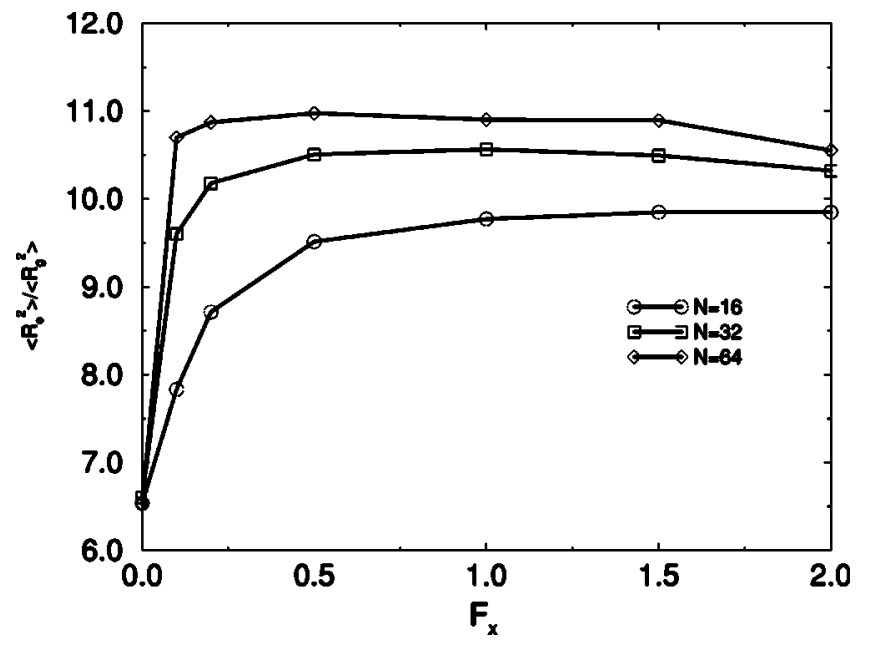

FIG. 5. $\left\langle R_{e}^{2}\right\rangle /\left\langle R_{g}^{2}\right\rangle$ as a function of external field $F_{x}$ for for chain lengths $N=16,32$, and 64 at $\rho_{i m p}=0.2$.

the chain is mostly stretched. For a given chain, an estimate of $F_{x}$ could be obtained from the dimensionless quantity $F_{x} R_{g} / k_{B} T$. We find that for low $\rho_{i m p}$ the chain stretching reaches a saturation for $F_{x} R_{g} / k_{B} T \sim 1$. Moreover for $\rho_{i m p}$ $=0.2$ the average pore spacing $r_{0}$ is $\sim 1.2$, while the radii of gyration for $\mathrm{N}=16,32$, and 64 are $\sim 2.5,5.0$, and 12.0, respectively which are larger than $r_{0}$. Therefore, the ratio $r_{0} / R_{g}$ dictates the minimum value of $F_{x}$ for which the chain is mostly stretched.

We now look at the longitudinal and the transverse sizes separately to get a better idea about the conformational properties of the chain. Since the chain is stretched along the direction of the bias, it is worthwhile to compare the transverse and the longitudinal extensions of the chain. Figure 6 shows the qualitative feature of the longitudinal and the transverse extensions of the chains as a function of the bias keeping $\rho_{i m p}$ as a parameter. For moderate densities of the impurities and low bias, evidently, the chain is not only

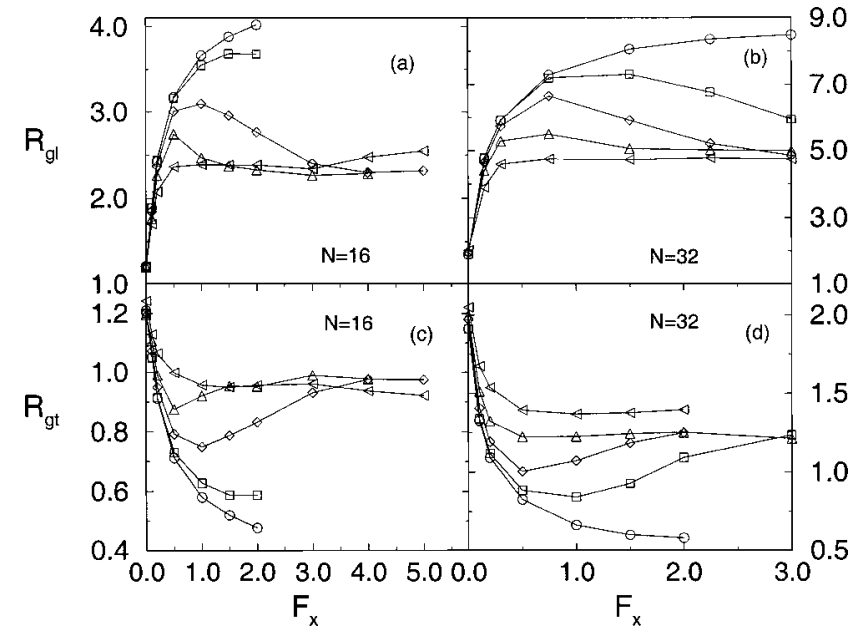

FIG. 6. $\left\langle R_{g l}\right\rangle$ [Figs. 5(a) and 5(b)] and $\left\langle R_{g t}\right\rangle$ [Figs. 5(c) and 5(d)] as a function of the bias $F_{x}$ for different values of the impurity density $\rho_{i m p}$; for chain the length $N=16[$ (a) and (c)] and for the chain length $N=32[(\mathrm{~b})$ and (d)]. The symbols $\bigcirc, \square, \diamond, \triangle, \triangleleft$ correspond to $\rho_{i m p}=0.1,0.2,0.3,0.4$, and 0.5 , respectively.

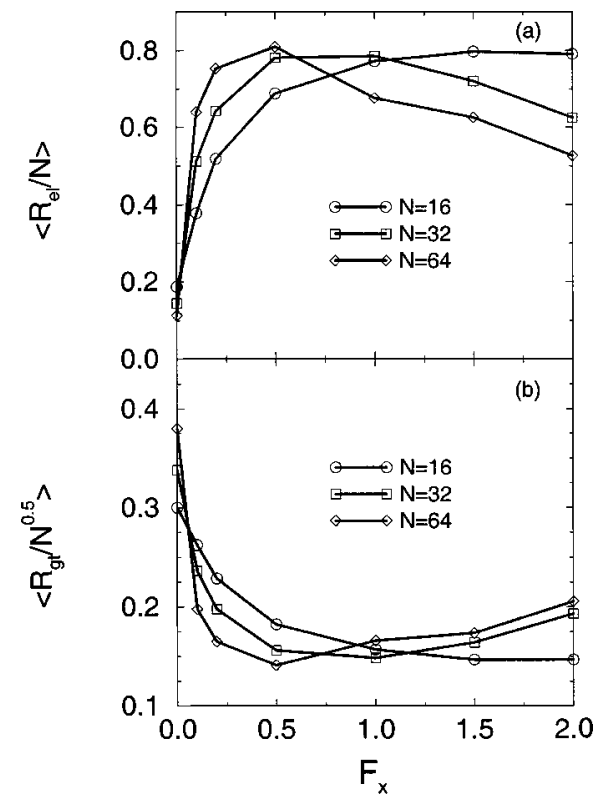

FIG. 7. $\left\langle R_{e l}\right\rangle / N$ and $\left\langle R_{g t}\right\rangle / \sqrt{N}$ as a function of the bias $F_{x}$ for chain lengths $N=16,32$, and 64 at $\rho_{\text {imp }}=0.2$.

stretched along the direction of the bias but shrinks in the transverse direction. However, with the increasing bias and the impurity density, a saturation effect is observed while with a further increase in the bias the effect is reversed. For the case when no impurities are present it is easy to show that for the longitudinal component of the end-to-end distance $\left\langle R_{e l}\right\rangle \simeq\left(\left\langle R_{g}^{2}\right\rangle / k_{B} T\right) F_{x}$ and $\left\langle R_{e l}\right\rangle=N l\left(F_{x} l / k_{B} T\right)^{2 / 3}$ for small and large tensions, respectively [3]. Similarly it can be shown that the transverse component of the radius of gyration $\left\langle R_{g t}^{2}\right\rangle \sim N l^{2}\left(k_{B} T / F_{x} l\right)^{1 / 3}$. Figures 7(a) and 7(b) show the plots of $\left\langle R_{e l}\right\rangle / N$ and $\left\langle R_{g t}\right\rangle / \sqrt{N}$ for different chain lengths as a function of the bias. They exhibit maxima/minima at different values of $\rho_{i m p}$ for different chain lengths. Evidently, in presence of the impurities, the simple scaling laws for $\left\langle R_{e l}\right\rangle$ and $\left\langle R_{e t}\right\rangle$ are not observed in our simulation. A proper scaling analysis in presence of the impurities, would require the ratio $r_{0} /\left\langle R_{g}\right\rangle$ to be taken in to account as an additional dimensionless variable as mentioned in the previous paragraph. However, it is worth noticing that the effect of the impurity and the bias becomes more drastic with the increasing chain lengths and the computational effort for averaging over sufficiently many runs and different impurity configurations becomes a problem even for modern computer facilities.

\section{Dynamic properties of the chain}

We have monitored the average rms displacement $X_{c . m .}^{2}(t)$ and the velocity of the center of mass of the chain $V_{d}=\dot{X}_{c . m}$. along the direction of the force as a function of both $F_{x}$ and $\rho_{i m p}$. For low $\rho_{i m p}$, the velocity increases linearly with the increasing bias, as expected. However, the velocity exhibits a maximum for intermediate values of concentrations as shown in Fig. 8, where we have shown the variation of the drift velocity for several values of the impurity density $\rho_{i m p}$ for the chains of lengths 8,16 , and 32, respectively. Figure 9 explicitly shows the length dependence 


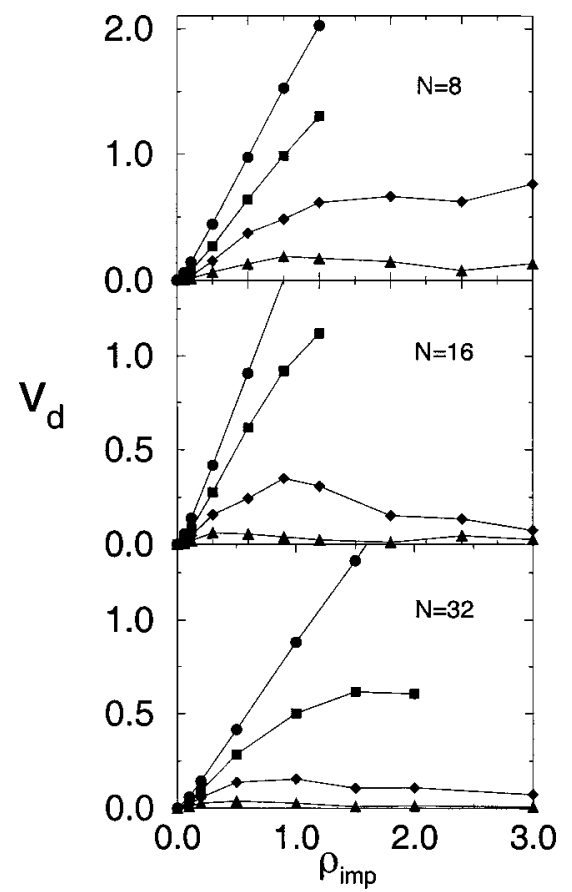

FIG. 8. $V_{d}$ as a function of the bias $F_{x}$ for for chain lengths $N$ $=8,16$, and 32 for different values of $\rho_{\text {imp }}$. Filled $\bigcirc, \square, \diamond$, and $\triangle$ correspond to $\rho_{i m p}=0.1,0.2,0.3$, and 0.4 , respectively.

for a given impurity density $\rho_{i m p}=0.2$. From Fig. 9 it appears that the critical bias $F_{x}^{\text {crit }} \sim 1 / N$ for $\rho_{\text {imp }}=0.2$. Occasionally, at large bias, the chain can overcome the entropic barrier and access regions of high entropic penalty from which it becomes difficult to get out which explains its reduced drift velocity. If we compare Fig. 9 with Fig. 5, we notice that the ratios for different chain lengths beyond $F_{x}^{\text {crit }}$ are significantly larger than 6 which indicate that, for a large bias, the chain does not have sufficient time to meander its way around the obstacles and encounters the repulsive barrier of the obstacle as happens in a "head-on" collision more frequently. The rest of the chain, being neutral, samples the available volume which is consistent with the fact that the

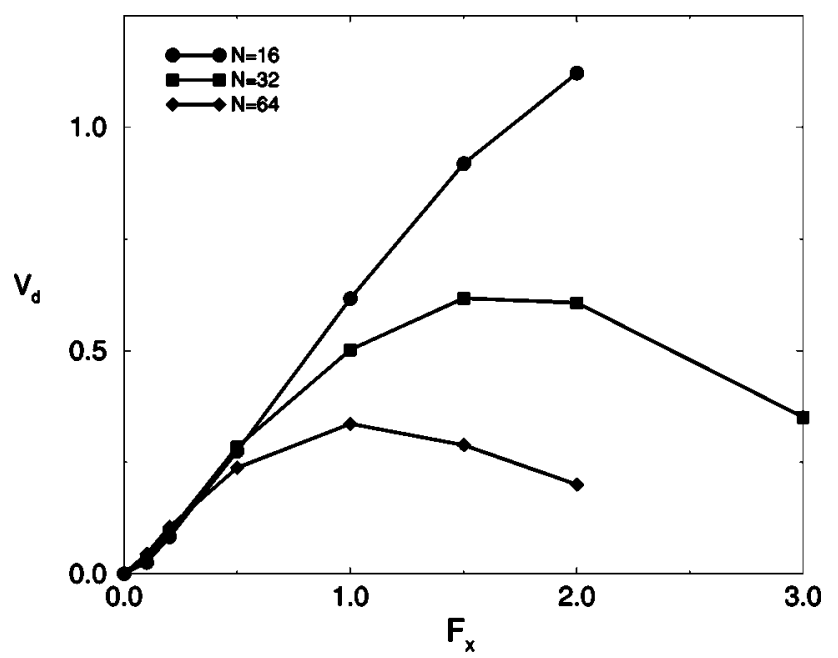

FIG. 9. $V_{d}$ as a function of the bias $F_{x}$ for for chain lengths $N$ $=16,32$, and 64 at $\rho_{i m p}=0.2$.
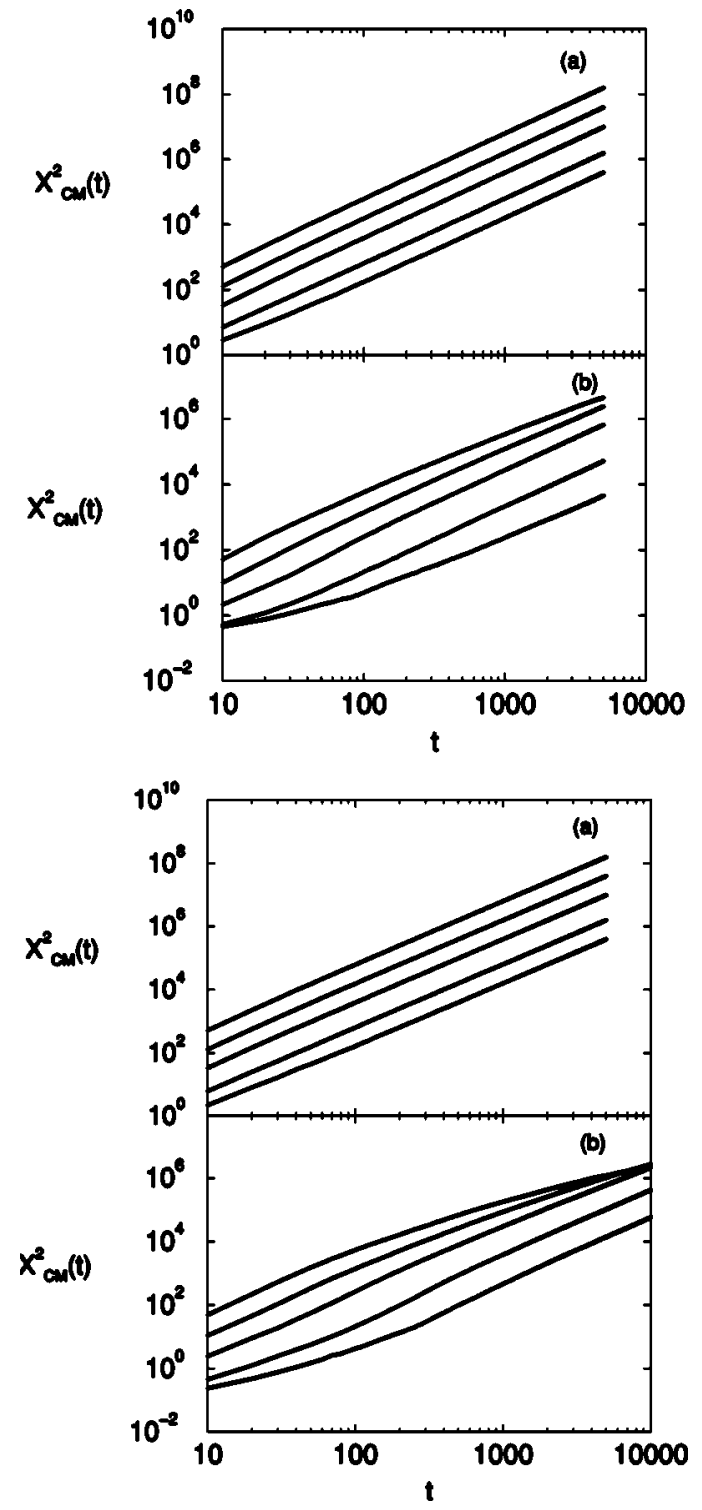

FIG. 10. Log-log plot of $X_{c m}^{2}(t)$ as a function of time for chain lengths $N=16$ (left) and 32 (right) for $\rho_{\text {imp }}=0.1$ and 0.2 , respectively. In each figure the bias increases from the bottom to the top.

Flory exponent is $\geqslant 0.6$. The reduced drift velocity is also manifested in the observed deviation from linearity in the $\log -\log$ plot of the $X_{c . m .}^{2}(t)$ as a function of $t$ as shown in Fig. 10 . For low impurity density $X_{c . m .}(t) \sim V_{d}\left(\rho_{\text {imp }}, F_{x}\right) t$, therefore, the slope of the straight lines is 2 . But we notice a deviation from linearity for the chain length $N=32$ and for $\rho_{\text {imp }}=0.2$ (right bottom) for larger biases.

\section{DISCUSSION}

In summary, we have investigated both the static and the dynamic properties of an end-labeled telechelic polymer chain in presence of quenched random impurities and driven by an external field using a stochastic MD simulation method. Our numerical data shows a minimum in $\left\langle R_{g}\right\rangle$ with increasing density of randomness. The trend suggests that for very long chains this effect will disappear as the position of 
the minimum shifts toward $\rho_{\text {imp }} \rightarrow 0$ for longer chains. Further systematic runs with smaller densities and longer chain will settle this issue.

In absence of a bias, our MD data reveals several important results. For the impurity densities $\rho_{\text {imp }}=0.0-0.6$, our simulation results show that the effective Flory exponent $\nu_{\text {eff }} \geqslant 0.6$, which indicates that the conformation of the chain at high impurity density is still described by a self avoiding random walk (albeit with the "persistent length" of the medium, as proposed in a paper by Panyukov. This has also been observed previously in MC calculation [13]. This is an important result and probably indicative of the limitations of the variational calculations using the replica trick as it predicts otherwise.

The effect of the bias is more drastic for the longer chains. Therefore, it seems plausible that in the limit of very long chains and in presence of a weak disorder, an extremely small but nonzero bias would be sufficient to stretch the chain completely. From our simulation data it appears that in presence of disorder the simple scaling laws for the transverse and the longitudinal components are violated. A more refined scaling analysis would require an obvious additional variable $r_{0} /\left\langle R_{g}\right\rangle$.

The observed maximum for the drift velocity as a function of the bias is consistent with the idea of jamming. Once the telechelic head gets stuck in a narrow channel among obstacles and the large bias does not let it get loose, it can take very long time to escape out of that region which would explain the observed reduction of the drift velocity at large bias. Note that the neutral tail of the polymer does not help the immobile head in this situation. It will be worthwhile to calculate the residence time for such event as a function of the chain length, randomness and the bias. Presently we are extending our calculations for larger chain lengths and for more numerous biases and impurity densities. In particular the intermediate density regime, where both the static and the dynamic properties exhibit extrema, are worth investigating in more detail. Our simulation does not take into account any hydrodynamic effects which may also play a role, however, one expects that at small free volume in the system hydrodynamic corrections will be small. The most straightforward method to include hydrodynamic effects in a computer experiment is to simulate explicitly all molecules of the solvent in addition to those of the polymer chains. As the solvent particles are much smaller than the monomers, and comprise a dense fluid themselves, such simulation would require prohibitively long time and is nowadays hardly manageable. A smarter method which allows for hydrodynamic effects albeit without considering the solvent particles explicitly, has been recently introduced by combining the Lattice Boltzmann method and MD [26]. It is worth exploring this new method in the present context for low and inetermediate desities of the obstacles. The drift of the chain in various regular and periodic geometries may also reveals important differences when compared with the results reported in this paper. Furthermore, the MD approach will be very desirable to take into account a dynamic environment of the obstacles. Some of these issues are currently under investigation and will be reported in a separate publication.

\section{ACKNOWLEDGMENTS}

This work is partially supported by the National Science Foundation (ENG/ECS and CISE/EIA) under Grant No. $0103587(\mathrm{AB})$ and partial support for computation at UCF. Andrey Milchev acknowledges the hospitality and support from UCF.
[1] J. Viovy, Rev. Mod. Phys. 72, 813 (2000).

[2] A. Baumgartner and M. Muthukumar, Advances in Chemical Physics XCIV, edited by I. Prgogine and S. A. Rice (Wiley, New York, 1996).

[3] P. G. de Gennes, Scaling Concepts in Polymer Physics (Cornell University Press, Ithaca, New York, 1979).

[4] M. Doi and S. F. Edwards, The Theory of Polymer Dynamics (Oxford University Press, Oxford, 1986).

[5] S. Stepanow and M. Schultz, Europhys. Lett. 47, 643 (1999).

[6] A. Baumgartner and M. Muthukumar, J. Chem. Phys. 87, 3082 (1987).

[7] S.F. Edwards and M. Muthukumar, J. Chem. Phys. 89, 2435 (1988)

[8] M. Muthukumar, J. Chem. Phys. 90, 4594 (1989).

[9] M. Muthukumar and A. Baumgartner, Macromolecules 22, 1941 (1989).

[10] M. Muthukumar and A. Baumgartner, Macromolecules 22, 1937 (1989).

[11] S.V. Panyukov, Zh. Eksp. Teor. Fiz. 103, 1287 (1993) [JETP 76, 631 (1993)].

[12] V. Yamakov and A. Milchev, Phys. Rev. E 56, 7043 (1997).

[13] V. Yamakov and A. Milchev, Phys. Rev. E 55, 1704 (1997).
[14] S.Y. Wu and G.W. Slater, Macromolecules 26, 1905 (1993).

[15] K. Avramova and A. Milchev, Eur. Phys. J. E 7, 65 (2002).

[16] G.M. Foo and R.B. Pandey, Phys. Rev. E 51, 5738 (1995).

[17] G.M. Foo and R.B. Pandey, J. Chem. Phys. 112, 10659 (2000).

[18] P. Pincus, Macromolecules 9, 386 (1976).

[19] K. Kremer and G.S. Grest, J. Chem. Phys. 92, 5057 (1990); K. Kremer and G.S. Grest, in Monte Carlo and Molecular Dynamics Simulations in Polymer Science, edited by K. Binder (Oxford University Press, Oxford, 1995); G.S. Grest and M. Murat, ibid.

[20] Aniket Bhattacharya, S.D. Mahanti, and Amitabha Chakrabarti, Phys. Rev. Lett. 80, 333 (1998).

[21] W.F. van Gunsteren and H.J.C. Berendsen, Mol. Phys. 45, 637 (1982).

[22] S. Chandrasekhar, Rev. Mod. Phys. 15, 1 (1943).

[23] R. Toral and A. Chakrabarti, Comput. Phys. Commun. 74, 327 (1993).

[24] G.S. Grest, B. Dunweg, and K. Kremer, Comput. Phys. Commun. 55, 269 (1989).

[25] M. Allen and D. Tildesley, Computer Simulation of Liquids (Clarendon Press, Oxford, 1987).

[26] P. Allrichs and B. Dünweg, J. Chem. Phys. 111, 8225 (1999). 